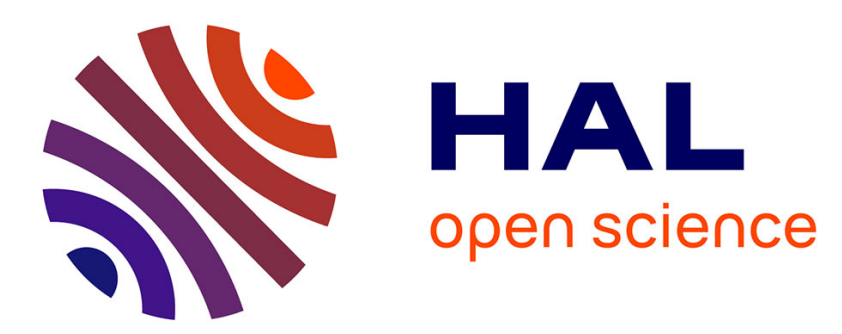

\title{
Mode detection and discernability as a framework for the estimation of time-varying delays
}

\author{
Meriem Halimi, Gilles Millérioux, Jamal Daafouz
}

\section{To cite this version:}

Meriem Halimi, Gilles Millérioux, Jamal Daafouz. Mode detection and discernability as a framework for the estimation of time-varying delays. 13th European Control Conference, ECC'14, Jun 2014, Strasbourg, France. hal-01079923

\section{HAL Id: hal-01079923 \\ https://hal.science/hal-01079923}

Submitted on 25 Nov 2014

HAL is a multi-disciplinary open access archive for the deposit and dissemination of scientific research documents, whether they are published or not. The documents may come from teaching and research institutions in France or abroad, or from public or private research centers.
L'archive ouverte pluridisciplinaire HAL, est destinée au dépôt et à la diffusion de documents scientifiques de niveau recherche, publiés ou non, émanant des établissements d'enseignement et de recherche français ou étrangers, des laboratoires publics ou privés. 


\title{
Mode detection and discernability as a framework for the estimation of time-varying delays
}

\author{
Mereim Halimi $^{1,2}$ and Gilles Millérioux ${ }^{1,2}$ and Jamal Daafouz ${ }^{1,2}$
}

\begin{abstract}
In this paper, it is shown how the issue of reconstructing time-varying unknown delays for linear systems can be turned into a mode detection one for switched affine systems. The specificities due to this reformulation are highlighted. The property of discernability, that is the ability for a detector to deliver a unique solution, is specifically addressed. An example is given as an illustration of the efficiency of the approach.
\end{abstract}

Time-delay systems have been the subject of intensive researches over the years. This is due to the fact that time delays are inherent in many real physical systems, such as mechanical systems, chemical processes, biology, transportation or communication systems and econometric models. The estimation of time delays of dynamical systems has received considerable attention in automatic control. Although a review and a comparison of time-varying delays estimation approaches have been proposed in the literature, see [8] and more recently [7], most of the available methods are dedicated to continuous systems. The problem of the estimation of the delay for discrete-time systems is rather scarce and motivates the present work. It is shown that such an issue for linear systems can be turned into a special mode detection one for switched affine systems.

An important concept for mode detection purpose is the so-called discernability. Roughly speaking, discernability reflects the ability of discriminating the active sequence from any other ones. In other words, it deals with the matter of uniqueness. A sufficient condition for discernability to hold is the observability [13]. However, less restrictive conditions can be found in the literature like $(\eta, \omega)$-discernability [1], Backward Discernability and Forward Discernability [3]. For systems with input, discernability deserves a special treatment because it depends on the input sequence. In this respect, the so-called active mode observation has been addressed in [2], [4] and [5] where a distinction between discerning control sequences over a finite or an infinite horizon of time is made. In this paper, discernability is tackled in the special context of the estimation of timevarying delays.

This paper is organized as follows. Section I is devoted to the problem statement. The reformulation of the estimation of time-varying delays as a mode detection problem for switched affine systems is carried out. In section II, discern-

\footnotetext{
1 Université de Lorraine, CRAN, UMR 7039, ESSTIN, 2 rue Jean Lamour, Vandouvre-lès-Nancy Cedex, 54506, France

2 CNRS, CRAN, UMR 7039, France
}

ability is discussed in the general context. It is introduced the notion of $(\lambda)$-discernability. In Section III, discernability and mode detection methodologies are particularized for the purpose of time-varying delays estimation. The paper ends up with an illustrative example in Section IV.

Notation: $\mathbb{N}$ is the set of natural numbers. $\mathbb{R}^{n}$ is the $n$ dimensional set of real numbers. $\mathbf{1}$ is the identity matrix and $\mathbf{0}$ is the zero matrix, being both of appropriate dimension when unspecified. $X^{T}$ is the transpose of the matrix $X$. The matrix $X^{\dagger}$ stands for the generalized inverse (Moore-Penrose) of $X$ satisfying $X^{\dagger} X$ symmetric, $X X^{\dagger}$ symmetric, $X X^{\dagger} X=X$ and $X^{\dagger} X X^{\dagger}=X^{\dagger}$. The kernel (null space) of $X$ is denoted $\operatorname{ker}(X) \cdot \mathcal{R}(X)$ is the column range space of $X$. The rank of a matrix $X$ is denoted $\operatorname{rank}(X)$.

\section{Problem STATEMENT}

Let us consider the discrete-time affine delayed system given by

$$
\left\{\begin{aligned}
X_{k+1} & =\mathcal{A} X_{k}+\mathcal{G} X_{k-\tau(k)}+\mathcal{B} U_{k-\tau^{\prime}(k)}+\mathcal{E} \\
Y_{k} & =\mathcal{C} X_{k}+\mathcal{D} U_{k}
\end{aligned}\right.
$$

where $k$ is the natural number standing for the discretetime, $X_{k} \in \mathbb{R}^{N}$ is the state vector, $Y_{k} \in \mathbb{R}^{M}$ is the output, $U_{k} \in \mathbb{R}^{P}$ is the input. The matrices $\mathcal{A} \in \mathbb{R}^{N \times N}$, $\mathcal{G} \in \mathbb{R}^{N \times N}, \mathcal{B} \in \mathbb{R}^{N \times P}, \mathcal{C} \in \mathbb{R}^{M \times N}, \mathcal{D} \in \mathbb{R}^{M \times P}$ and $\mathcal{E} \in \mathbb{R}^{N \times 1}$ are constant matrices. The quantities $\tau(k)$ and $\tau^{\prime}(k)$ are time-varying delays acting respectively on the state vector and on the input. They take values in two respective finite sets $\mathcal{T}=\{0,1, \cdots, \alpha\}$ and $\mathcal{T}^{\prime}=\left\{0,1, \cdots, \alpha^{\prime}\right\}$. The time variation of $\tau(k)$ and $\tau^{\prime}(k)$ is arbitrary. The case when $\tau(k)$ and $\tau^{\prime}(k)$ are not directly accessible is considered. Hence, the aim is to estimate $\tau(k)$ and $\tau^{\prime}(k)$ while the model, the sequences $U_{k}$ and $Y_{k}$ are assumed to be known and available on-line.

As a clue to tackle this problem, we can interestingly point out that such an issue can be reformulated as a mode detection problem of an equivalent switched affine system. Indeed, despite a different purpose, the same lines of reasoning than the ones suggested in [11] [12] can be followed, that is, a switched system transformation approach can be used. It is detailed below.

Let $\sigma$ be a function $\sigma: \mathbb{N} \rightarrow \mathcal{J}$ which, at any time $k$, uniquely identify the pair $\left(\tau(k), \tau^{\prime}(k)\right) \in \mathcal{T} \times \mathcal{T}^{\prime}$ according to the following one-to-one correspondence: $\left(\tau(k), \tau^{\prime}(k)\right)=$ $(0,0) \leftrightarrow \sigma(k)=1,\left(\tau(k), \tau^{\prime}(k)\right)=(1,0) \leftrightarrow \sigma(k)=2, \ldots$, $\left(\tau(k), \tau^{\prime}(k)\right)=(\alpha, 0) \leftrightarrow \sigma(k)=\alpha+1,\left(\tau(k), \tau^{\prime}(k)\right)=$ 
$(0,1) \leftrightarrow \sigma(k)=\alpha+2, \ldots,\left(\tau(k), \tau^{\prime}(k)\right)=\left(0, \alpha^{\prime}\right) \leftrightarrow$ $\sigma(k)=(\alpha+1) \cdot \alpha^{\prime}+1, \ldots,\left(\tau(k), \tau^{\prime}(k)\right)=\left(\alpha, \alpha^{\prime}\right) \leftrightarrow$ $\sigma(k)=(\alpha+1) \cdot\left(\alpha^{\prime}+1\right)$. Then, defining

$$
x_{k}=\left[\begin{array}{c}
X_{k} \\
X_{k-1} \\
\vdots \\
X_{k-\alpha}
\end{array}\right], \quad u_{k}=\left[\begin{array}{c}
U_{k} \\
U_{k-1} \\
\vdots \\
U_{k-\alpha^{\prime}}
\end{array}\right], \quad y_{k}=Y_{k}
$$

The system (1) can be equivalently rewritten as a switching affine system like

$$
\left\{\begin{aligned}
x_{k+1} & =A_{\sigma(k)} x_{k}+B_{\sigma(k)} u_{k}+E_{\sigma(k)} \\
y_{k} & =C_{\sigma(k)} x_{k}+D_{\sigma(k)} u_{k}
\end{aligned}\right.
$$

where $x_{k} \in \mathbb{R}^{n}$ is the state vector with $n=(\alpha+1) N, y_{k} \in$ $\mathbb{R}^{m}$ is the output with $m=M$, and $u_{k} \in \mathbb{R}^{p}$ is the input with $p=\left(\alpha^{\prime}+1\right) P$. The function $\sigma$ defined above corresponds for (3) to the switching law $\sigma: \mathbb{N} \rightarrow \mathcal{J}=\{1, \ldots, J\}$ with $J=(\alpha+1)\left(\alpha^{\prime}+1\right)$ that assigns, to any discrete-time $k \in \mathbb{N}$, the integer $\sigma(k) \in \mathcal{J}$. The matrices $A_{\sigma(k)} \in \mathbb{R}^{n \times n}, B_{\sigma(k)} \in$ $\mathbb{R}^{n \times p}, C_{\sigma(k)} \in \mathbb{R}^{m \times n}, D_{\sigma(k)} \in \mathbb{R}^{m \times p}$ and $E_{\sigma(k)} \in \mathbb{R}^{n \times 1}$ are the state space matrices of the system which belong to the respective sets $\left\{A_{1}, \ldots, A_{J}\right\},\left\{B_{1}, \ldots, B_{J}\right\},\left\{C_{1}, \ldots, C_{J}\right\}$, $\left\{D_{1}, \ldots, D_{J}\right\}$ and $\left\{E_{1}, \ldots, E_{J}\right\}$. The state space matrices obey the following construction

$$
\begin{aligned}
A_{\sigma(k)}= & {\left[\begin{array}{cccc}
\mathcal{A}+\kappa(\tau(k)) \mathcal{G} & \psi_{1}(\tau(k)) & \cdots & \psi_{\alpha}(\tau(k)) \\
\mathbf{1} & \mathbf{0} & \cdots & \mathbf{0} \\
\mathbf{0} & \mathbf{1} & \cdots & \mathbf{0} \\
\vdots & & \cdots & \vdots \\
\mathbf{0} & \mathbf{0} & \cdots & \mathbf{0}
\end{array}\right] } \\
B_{\sigma(k)} & =\left[\begin{array}{cccc}
\psi_{0}^{\prime}\left(\tau^{\prime}(k)\right) & \psi_{1}^{\prime}\left(\tau^{\prime}(k)\right) & \cdots & \psi_{\alpha}^{\prime}\left(\tau^{\prime}(k)\right) \\
\mathbf{0} & \mathbf{0} & \cdots & \mathbf{0}
\end{array}\right] \\
E_{\sigma(k)}= & {\left[\begin{array}{c}
\mathcal{E} \\
\mathbf{0}
\end{array}\right], C_{\sigma(k)}=\left[\begin{array}{ll}
\mathcal{C} & \mathbf{0}
\end{array}\right], D_{\sigma(k)}=\left[\begin{array}{ll}
\mathcal{D} & \mathbf{0}
\end{array}\right] }
\end{aligned}
$$

$\psi_{i}$ is defined for $i=1, \ldots, \alpha$ as

$$
\psi_{i}(\tau(k))=\left\{\begin{array}{l}
\mathcal{G} \text { if } \tau(k)=i \\
\mathbf{0} \text { if } \tau(k) \neq i
\end{array}, \kappa(\tau(k))=\left\{\begin{array}{l}
1 \text { if } \tau(k)=0 \\
0 \text { if } \tau(k) \neq 0
\end{array}\right.\right.
$$

Finally, $\psi_{i}^{\prime}$ is defined for $i=0, \ldots, \alpha$ as

$$
\psi_{i}^{\prime}\left(\tau^{\prime}(k)\right)=\left\{\begin{array}{lll}
\mathcal{B} & \text { if } & \tau^{\prime}(k)=i \\
\mathbf{0} & \text { if } & \tau^{\prime}(k) \neq i
\end{array}\right.
$$

This being the case, estimating $\tau(k)$ and $\tau^{\prime}(k)$ of (1) amounts to estimate the mode $\sigma(k)$ of (3), being known a sequence of input/output data $u_{k}$ and $y_{k}$ over a finite length receding horizon. As a result, we can write without any confusion a sequence of modes $\sigma_{s}=\sigma(k) \sigma(k+1) \ldots \sigma(k+h)$ and a sequence of pairs of delays $\sigma_{s}=\left(\tau(k), \tau^{\prime}(k)\right)(\tau(k+$ $\left.1), \tau^{\prime}(k+1)\right) \ldots\left(\tau(k+h), \tau^{\prime}(k+h)\right)$. Hereafter, $\sigma_{\left[k_{1}, k_{2}\right]}$ will stand for the finite sequence of modes (also called path) $\left\{\sigma\left(k_{1}\right), \cdots, \sigma\left(k_{2}\right)\right\}$ in the interval of time $\left[k_{1}, k_{2}\right]$. We denote with $\mathcal{S}$ the set of integers $s \in \mathcal{S}$ that uniquely identify a sequence $\sigma_{s} \in \mathcal{J}^{h+1}$ in the interval of time $[k-h, k]$ and with $S$ the number of admissible sequences in $\mathcal{J}^{h+1}$. By admissible sequence, it is meant a sequence for which $\sigma(k+1)$ is compatible with $\sigma(k)$ and the transition rule defined by the switching function $\sigma$. The active sequence will be denoted with $\sigma^{*}$.

Hence, we can resort to the mode detection proposed in [9] and recalled in the following proposition.

Proposition 1: [9] Let $h$ be a horizon length. The active sequence $\sigma^{*}$ in the interval of time $[k-h, k]$ is a sequence $\sigma_{s} \in \mathcal{J}^{h+1}$ such that the corresponding residual fulfills

$$
r_{h, \sigma_{s}}=\mathbf{0}
$$

with

$$
r_{h, \sigma_{s}}=\Omega_{\sigma_{s}}\left(y_{k-h, k}-T_{\sigma_{s}} u_{k-h, k}-T_{\sigma_{s}}^{\prime}\right)
$$

where $\Omega_{\sigma_{s}}$ is the solution of $\Omega_{\sigma_{s}} \mathcal{O}_{\sigma_{s}}=\mathbf{0}$, with

$$
y_{k-h, k}=\mathcal{O}_{\sigma_{[k-h, k]}} x_{k-h}+T_{\sigma_{[k-h, k]}} u_{k-h, k}+T_{\sigma_{[k-h, k]}}^{\prime}
$$

where

$$
\begin{aligned}
& \mathcal{O}_{\sigma_{[k-h, k]}}=\left[\begin{array}{c}
C_{\sigma(k-h)} \\
C_{\sigma(k-h+1)} A_{\sigma(k-h)} \\
\vdots \\
C_{\sigma(k-1)} A_{\sigma(k-2)}^{\sigma(k-h)} \\
C_{\sigma(k)} A_{\sigma(k-1)}^{\sigma(k-h)}
\end{array}\right] \\
& T_{\sigma_{[k-h, k]}}=\left[\begin{array}{ccc}
D_{\sigma(k-h)} & \mathbf{0} & \mathbf{0} \\
C_{\sigma(k-h+1)} B_{\sigma(k-h)} & D_{\sigma(k-h+1)} & \mathbf{0} \\
\vdots & \ddots & \vdots \\
C_{\sigma(k-1)} B_{\sigma(k-2)}^{\sigma(k-h)} & D_{\sigma(k-1)} & \mathbf{0} \\
C_{\sigma(k)} B_{\sigma(k-1)}^{\sigma(k-h)} & & D_{\sigma(k)}
\end{array}\right] \\
& T_{\sigma_{[k-h, k]}}^{\prime}=\left[\begin{array}{c}
\mathbf{0} \\
C_{\sigma(k-h+1)} E_{\sigma(k-h)} \\
\vdots \\
C_{\sigma(k-1)} E_{\sigma(k-2)}^{\sigma(k-h)} \\
C_{\sigma(k)} E_{\sigma(k-h)}^{\sigma(k-1)}
\end{array}\right] \\
& A_{k_{1}}^{k_{2}}=A_{k_{1}} A_{k_{1}+1} \cdots A_{k_{2}} \\
& B_{k_{1}}^{k_{2}}=\left[\begin{array}{llllll}
A_{k_{1}}^{k_{2}-1} B_{k_{2}} & \cdots & A_{k_{1}}^{k_{1}+1} B_{k_{1}+2} & A_{k_{1}} B_{k_{1}+1} & B_{k_{1}}
\end{array}\right] \\
& E_{k_{1}}^{k_{2}}=A_{k_{1}}^{k_{2}-1} E_{k_{2}}+\cdots+A_{k_{1}}^{k_{1}+1} E_{k_{1}+2}+A_{k_{1}} E_{k_{1}+1}+E_{k_{1}} \\
& y_{k_{1}, k_{2}}=\left[\begin{array}{llll}
y_{k_{1}}^{T} & y_{k_{1}+1}^{T} & \cdots & y_{k_{2}}^{T}
\end{array}\right]^{T} \\
& u_{k_{1}, k_{2}}=\left[\begin{array}{llll}
u_{k_{1}}^{T} & u_{k_{1}+1}^{T} & \cdots & u_{k_{2}}^{T}
\end{array}\right]^{T}
\end{aligned}
$$

$\mathcal{O}_{\sigma_{[k-h, k]}}$ is the observability matrix in the finite observation window $[k-h, k]$.

Actually, the horizon length $h$ is defined in [9] as the integer which satisfies $\operatorname{rank}\left(\mathcal{O}_{\sigma_{[k-h, k]}}\right)=n$. That supposes the system to be pathwise observable and is restrictive. It has been shown in [10] that such a condition can be relaxed and $h$ must obey

$$
\operatorname{rank}\left(\mathcal{O}_{\sigma_{[k-h, k]}}\right)=\operatorname{rank}\left(\mathcal{O}_{\sigma_{[k-h, k-1]}}\right)
$$

An important issue to be addressed for mode detection purpose is the ability for a detector to deliver a unique solution, that is a unique mode sequence $\sigma_{s}$. Let us stress 
that the detector defined as in Proposition 1 does not exclusively deliver the active mode $\sigma(k)$ at time $k$ but deliver the whole active sequence $\sigma^{*}$ in the interval of time $[k-h, k]$. Addressing the question of uniqueness amounts to checking whether the mode detector is able to discriminate the active sequence $\sigma^{*}$ from any other sequences $\sigma_{s} \in \mathcal{J}^{h+1}$. Such a notion is related to the so-called discernability.

First, we recall and provide new results on the question of uniqueness in the general case. Then, we particularize the investigation in the special context of time-varying delays estimation.

\section{UNIQUENESS}

\section{A. Discernability}

Discernability has been thoroughly discussed in [3] and [9]. Let us point out that some authors use the terminology "mode observability" instead of "discernability". Formally, discernability of mode sequences obeys the following definition which is restated here to be in accordance with the notation of the present paper and with the detector defined as in Proposition 1.

Definition 1: For the detector defined as in Proposition 1 based on the residual $r_{h, \sigma_{s}}$, two modes sequences $\sigma_{s_{1}}$ and $\sigma_{s_{2}}$ delivered by (3), with $\left(s_{1}, s_{2}\right) \in \mathcal{S}^{2}$, are discernible on an observation window of length $h+1$, if the corresponding residuals $r_{h, \sigma_{s_{1}}}$ et $r_{h, \sigma_{s_{2}}}$ are not simultaneously zero when one of the sequence $\sigma_{s_{1}}$ or $\sigma_{s_{2}}$ is the active sequence $\sigma^{*}$.

A necessary and sufficient condition for discernability of two sequences is proved in [9] and is recalled below.

Theorem 1: [9] For a given input sequence $u_{k-h, k}$, two mode sequences $\sigma_{s_{1}}$ and $\sigma_{s_{2}}$ delivered by (3), with $\left(s_{1}, s_{2}\right) \in \mathcal{S}^{2}$, are discernible on an observation window of length $h+1$, for almost all initial conditions $x_{k-h}$, iff at least one of the two following conditions is satisfied for all $i, j \in\left\{s_{1}, s_{2}\right\}, \quad i \neq j$

$$
\begin{gathered}
\Omega_{\sigma_{i}} \mathcal{O}_{\sigma_{j}} \neq \mathbf{0} \\
\Omega_{\sigma_{i}}\left(\left(T_{\sigma_{j}}-T_{\sigma_{i}}\right) u_{k-h, k}+\left(T_{\sigma_{j}}^{\prime}-T_{\sigma_{i}}^{\prime}\right)\right) \neq \mathbf{0}
\end{gathered}
$$

Finally, we can introduce the notion of discernability for (3).

Definition 2: For a given input sequence $u_{k-h, k}$, system (3) is discernible if, for every pairs of sequences $\sigma_{s_{1}}, \sigma_{s_{2}} \in \mathcal{J}^{h+1}$ with $\left(s_{1}, s_{2}\right) \in \mathcal{S}^{2}, \sigma_{s_{1}}$ is discernible from $\sigma_{s_{2}}$.

According to Definition 1, if discernability is not satisfied, this means that several residuals can vanish simultaneously. That causes the estimation of the sequence $\sigma_{s}$ provided in Proposition 1 to be unsuccessful. In other words, the active sequence of length $h+1$ cannot be discriminated from all the other ones and then, cannot be directly estimated. This being the case, an alternative to circumvent the problem has been proposed in the literature through the concept of $(\eta, \omega)-$ discernability [1]. It is the purpose of next subsection.

\section{B. $(\eta, \omega)$-Discernability}

The property of $(\eta, \omega)$-discernability has been introduced in [1]. It reflects the ability to discriminate, and then to estimate, the active subsequence $\sigma_{[k-h+\eta, k-\omega]}^{*}$ from all the other ones. The quantities $\eta$ and $\omega$ are positive integers verifying $\eta \in\{0, \ldots, h\}, \omega \in\{0, \ldots, h\}$ and $\eta+\omega \leq h$. Clearly, $(\eta, \omega)$-discernability coincides with discernability if $\eta=0$ and $\omega=0$. In the sequel, we shall consider either $\eta>0$ or $\omega>0$. The integers $\eta$ and $\omega$ shall be chosen as small as possible to get a estimated sequence of maximal length. Letting $\sigma_{s[k-h+\eta, k-\omega]}$ with $s \in \mathcal{S}$ as the sequence of modes $\sigma(k-h+\eta), \ldots, \sigma(k-\omega),(\eta, \omega)$-discernability obeys the following definition.

Definition 3: [1] $\sigma_{s_{1}}$ is $(\eta, \omega)$-discernible from $\sigma_{s_{2}}$ with $\sigma_{s_{1}}, \sigma_{s_{2}} \in \mathcal{J}^{h+1},\left(s_{1}, s_{2}\right) \in \mathcal{S}^{2}$, if $\sigma_{s_{1}[k-h+\eta, k-\omega]} \neq$ $\sigma_{s_{2}[k-h+\eta, k-\omega]}$ and $\sigma_{s_{1}}$ is discernible from $\sigma_{s_{2}}$.

Based on Theorem 1 of discernability, we can derive conditions for checking $(\eta, \omega)$-discernability as given in the following proposition.

Proposition 2: For a given input sequence $u_{k-h, k}$, two modes sequences $\sigma_{s_{1}}$ and $\sigma_{s_{2}}$ of (3) with $\left(s_{1}, s_{2}\right) \in \mathcal{S}^{2}$ are $(\eta, \omega)$-discernible on an observation window of length $h+1$, for almost all initial conditions $x_{k-h}$, iff at least one of the two following conditions is satisfied for all $i, j \in$ $\left\{s_{1}, s_{2}\right\}$ with $i \neq j$

$$
\begin{gathered}
\Omega_{\sigma_{i}} \mathcal{O}_{\sigma_{j}} \neq \mathbf{0} \\
\Omega_{\sigma_{i}}\left(\left(T_{\sigma_{j}}-T_{\sigma_{i}}\right) u_{k-h, k}+\left(T_{\sigma_{j}}^{\prime}-T_{\sigma_{i}}^{\prime}\right)\right) \neq \mathbf{0} \\
\text { with } \quad \sigma_{s_{1}[k-h+\eta, k-\omega]} \neq \sigma_{s_{2}[k-h+\eta, k-\omega]}
\end{gathered}
$$

Proof 1: The proof is a straightforward consequence of the consideration of both Definition 3 of $(\eta, \omega)-$ discernability and (8)-(9) of Theorem 1.

Definition 4: [1] For a given input sequence $u_{k-h, k},(3)$ is $(\eta, \omega)$-discernible, if, for every pairs of sequences $\sigma_{s_{1}}, \sigma_{s_{2}} \in$ $\mathcal{J}^{h+1}$ with $\left(s_{1}, s_{2}\right) \in \mathcal{S}^{2}$ such that $\sigma_{s_{1}[k-h+\eta, k-\omega]} \neq$ $\sigma_{s_{2}[k-h+\eta, k-\omega]}, \sigma_{s_{1}}$ is discernible from $\sigma_{s_{2}}$.

The estimation of the active subsequence $\sigma^{*}{ }_{[k-h+\eta, k-\omega]}$ can be performed according to the following proposition.

Proposition 3: Assume that (3) is $(\eta, \omega)$-discernable. Let $\overline{\mathcal{S}} \subset \mathcal{S}$ be the set of integers $s \in \mathcal{S}$ such that all the sequences $\sigma_{s[k-h+\eta, k-\omega]}$ differ one another. The active subsequence $\sigma^{*}[k-h+\eta, k-\omega]$ in the interval of time $[k-h, k]$ is the unique subsequence $\sigma_{s[k-h+\eta, k-\omega]}$ of the sequences $\sigma_{s}$ with $s \in \overline{\mathcal{S}}$ such that the corresponding residual fulfills

$$
r_{h, \sigma_{s}}=\mathbf{0}
$$

with

$$
r_{h, \sigma_{s}}=\Omega_{\sigma_{s}}\left(y_{k-h, k}-T_{\sigma_{s}} u_{k-h, k}-T_{\sigma_{s}}^{\prime}\right)
$$

where $\Omega_{\sigma_{s}}$ is the solution of $\Omega_{\sigma_{s}} \mathcal{O}_{\sigma_{s}}=\mathbf{0}$.

Proof 2: The proof follows along the same lines of reasoning as in the proof of Proposition 1 except that $s \in \mathcal{S}$ is replaced by $s \in \overline{\mathcal{S}}$, that is, only the residuals for which the subsequences $\sigma_{s[k-h+\eta, k-\omega]}$ which differ from all the other ones are considered. 
If discernability is satisfied, the first active sequence $\sigma_{[0, h]}^{*}$ can be obtained. On the other hand, if $(\eta, \omega)$-discernability is satisfied, at time $k=h$, the active subsequence $\sigma_{[\eta, h-\omega]}^{*}$ can be estimated but by a sliding window approach, the initial active sequence can be complemented to obtain a sequence of any prescribed length. On the other hand, the sequence $\sigma_{[0, \eta-1]}^{*}$ cannot be estimated. All in all, Proposition 3 allows to achieve the estimation of all the modes $\sigma(k)$ for $k \geq 0$ or $k \geq \eta$ accordingly. However, the complexity of the estimation of the mode $\sigma(k)$ at time $k$, in terms of required amount of data and number of residuals to be calculated and analyzed, can be reduced. Indeed, the estimation of the mode $\sigma(k)$ at time $k$ can be performed taking into account that all the modes at times $k^{\prime}<k$ have already been estimated and so are assumed to be known. Such a consideration motivated the introduction of the concept of $(\lambda)$-Backward Discernability.

\section{C. ( $\lambda)$-Backward Discernability and permanent estimation}

The property of $(\lambda)$-Backward Discernability allows to deliver at time $k$, the mode $\sigma(k-\lambda)$, being the modes $\sigma(k-i)(1+\lambda \leq i \leq h)$ already estimated. The quantity $\lambda$ is an integer such that $\lambda \in\{1, \ldots, h\} .(\lambda)$-Backward Discernability stands as an extension of the so-called Backward Discernability introduced in [3]. Indeed, Backward Discernability corresponds to the case when $\lambda=0$. More formally, $(\lambda)$-Backward Discernability obeys the following definition.

Definition 5: A mode $i$ is $(\lambda)$-Backward Discernible from another mode $j$, if there exists an integer $\lambda$ such that, in an observation window $[k-h, k]$, for every sequence $\sigma^{h-\lambda}$ of length $h-\lambda$, and for any two sequences $\sigma_{1}^{\prime}$ and $\sigma_{2}^{\prime}$ of length $\lambda, \sigma^{h-\lambda} i \sigma_{1}^{\prime}$ is discernible from $\sigma^{h-\lambda} j \sigma_{2}^{\prime}$.

It is clear that discernability implies $(\lambda)$-Backward Discernability. Conditions for guaranteeing $(\lambda)$-Backward Discernability are provided in the following proposition.

Proposition 4: For a given input sequence $u_{k-h, k}$, two modes $i$ and $j$ are $(\lambda)$-Backward discernible on an observation window $[k-h, k]$ of length $h+1$, for almost all initial conditions $x_{k-h}$ iff, for every sequence $\sigma^{h-\lambda}$ of length $h-\lambda$ and for any two sequences $\sigma_{1}^{\prime}$ and $\sigma_{2}^{\prime}$ of length $\lambda$ delivered by (3), at least one of the following conditions are satisfied for all $i, j \in \mathcal{J}, \quad i \neq j$

$$
\begin{gathered}
\Omega_{\sigma^{h-\lambda} i \sigma_{1}^{\prime}} \mathcal{O}_{\sigma^{h-\lambda} j \sigma_{2}^{\prime}} \neq \mathbf{0} \\
\Omega_{\sigma^{h-\lambda} i \sigma_{1}^{\prime}}\left(\left(T_{\sigma^{h-\lambda} j \sigma_{2}^{\prime}}-T_{\sigma^{h-\lambda} i \sigma_{1}^{\prime}}\right) u_{k-h, k}\right. \\
\left.+\left(T_{\sigma^{h-\lambda} j \sigma_{2}^{\prime}}^{\prime}-T_{\sigma^{h-\lambda} i \sigma_{1}^{\prime}}^{\prime}\right)\right) \neq \mathbf{0}
\end{gathered}
$$

Proof 3: The proof is a straightforward consequence of the consideration of both Definition 5 of $(\lambda)$-Backward Discernability and (8)-(9) of Theorem 1.

Definition 6: For a given input sequence $u_{k-h, k}$, system (3) is $(\lambda)$-Backward Discernible if, for every pairs of modes $(i, j) \in \mathcal{J}^{2}$, the mode $i$ is $(\lambda)$-Backward Discernible from the mode $j$.
When $(\lambda)$-Backward Discernability is satisfied, the estimation of the mode $\sigma(k-\lambda)$ at time $k$ can be performed according to the following proposition.

Proposition 5: Assume that (3) is $(\lambda)$-Backward Discernable. Let $\sigma^{h-\lambda}$ be a sequence of length $h-\lambda$. Let $\overline{\mathcal{S}}^{\sigma^{h-\lambda}} \subset \mathcal{S}$ be the set of integers $s \in \mathcal{S}$ such that $\sigma^{h-\lambda} i \sigma^{\prime}$ differ one another for all for all $i \in \mathcal{J}$ and for any sequences $\sigma^{\prime}$ of length $\lambda$. The active mode $\sigma^{*}(k-\lambda)$ in the interval of time $[k-h, k]$ is the unique mode $\sigma(k-\lambda)$ of the sequence $\sigma_{s}$ with $s \in \overline{\mathcal{S}}^{\sigma^{h-\lambda}}$ such that the corresponding residual fulfills

$$
r_{h, \sigma_{s}}=\mathbf{0}
$$

with

$$
r_{h, \sigma_{s}}=\Omega_{\sigma_{s}}\left(y_{k-h, k}-T_{\sigma_{s}} u_{k-h, k}-T_{\sigma_{s}}^{\prime}\right),
$$

where $\Omega_{\sigma_{s}}$ is the solution of $\Omega_{\sigma_{s}} \mathcal{O}_{\sigma_{s}}=\mathbf{0}$.

Proof 4: The proof follows along the same lines of reasoning as in the proof of Proposition 1 except that $s \in \mathcal{S}$ is replaced by $s \in \overline{\mathcal{S}}^{\sigma^{h-\lambda}}$, that is, for a given subsequence $\sigma^{h-\lambda}$ of length $h-\lambda$, only the residuals for which the mode $\sigma(k-\lambda)$ differs one another are considered, the rest of the sequence of length $\lambda+1$ being disregarded.

The relevance of Proposition 5 lies in that only the residuals of the sequences $\sigma^{h-\lambda} i \sigma^{\prime}\left(\sigma^{\prime} \in \mathcal{J}^{\lambda}\right)$ with $\sigma^{h-\lambda} \in \mathcal{J}^{h-\lambda}$ resulting from the estimation at time $k-1$ have to be checked by the detectors. That allows to reduce the number of tests: $J^{\lambda+1}$ sequences instead of $J^{h+1}$ sequences. Let us recall that $\lambda \leq h$.

When $(\lambda)$-Backward Discernability is considered, a sequence of length respectively equal to $h-\lambda$ must be known. It has been previously stressed that $(\eta, \omega)$-discernability allows to estimate with Proposition 5 the active subsequence $\sigma_{[\eta, h-\omega]}^{*}$ and can be complemented by shifting in time the procedure until a sequence of length $h-\lambda$ is known. Then, we can proceed to the permanent estimation based on a receding horizon technique for $k>h+\omega+\eta-1$.

\section{SPECIFICITY FOR TIME-DELAYED SYSTEMS}

As previously stressed, because of the restriction due to the specific structure of the state space matrices of (3), it can be suspected that some of the properties of discernability are no longer satisfied. This is precisely shown in what follows. All the proofs are based on the search for particular sequences for which the properties are not satisfied.

1) Preliminary result: The following lemma is recalled from [3].

Lemma 1: Given a vector $Y^{\prime}$ and a matrix $\mathcal{O}$, being $X$ unknown, it holds that

$$
Y^{\prime} \in \mathcal{R}(\mathcal{O}) \Leftrightarrow \exists X \mid Y^{\prime}=\mathcal{O} X \Leftrightarrow\left(\mathcal{O O}^{\dagger}-\mathbf{1}\right) Y^{\prime}=\mathbf{0}
$$

Proposition 6: Condition $\Omega_{\sigma_{s_{2}}} \mathcal{O}_{\sigma_{s_{1}}} \neq \mathbf{0}$ is equivalent to

$$
\operatorname{rank}\left(\left[\begin{array}{ll}
\mathcal{O}_{\sigma_{s_{1}}} & \mathcal{O}_{\sigma_{s_{2}}}
\end{array}\right]\right)>\operatorname{rank}\left(\mathcal{O}_{\sigma_{s_{2}}}\right)
$$


where $\left[\begin{array}{ll}\mathcal{O}_{\sigma_{s_{1}}} & \mathcal{O}_{\sigma_{s_{2}}}\end{array}\right]$ denotes the horizontal concatenation of $\mathcal{O}_{\sigma_{s_{1}}}$ and $\mathcal{O}_{\sigma_{s_{2}}}$.

Proof 5: If (17) is not satisfied, it is equivalent to $\operatorname{rank}\left(\left[\begin{array}{ll}\mathcal{O}_{\sigma_{s_{1}}} & \mathcal{O}_{\sigma_{s_{2}}}\end{array}\right]\right)=\operatorname{rank}\left(\mathcal{O}_{\sigma_{s_{2}}}\right)$ since it is clear that $\operatorname{rank}\left(\left[\begin{array}{ll}\mathcal{O}_{\sigma_{s_{1}}} & \mathcal{O}_{\sigma_{s_{2}}}\end{array}\right]\right)<\operatorname{rank}\left(\mathcal{O}_{\sigma_{s_{2}}}\right)$ can never occur. This means that $\mathcal{O}_{\sigma_{s_{1}}} \in \mathcal{R}\left(\mathcal{O}_{\sigma_{s_{2}}}\right)$. From (16) of Lemma 1, we get that

$$
\mathcal{O}_{\sigma_{s_{1}}} \in \mathcal{R}\left(\mathcal{O}_{\sigma_{s_{2}}}\right) \Leftrightarrow\left(\mathcal{O}_{\sigma_{s_{2}}} \mathcal{O}_{\sigma_{s_{2}}}^{\dagger}-\mathbf{1}\right) \mathcal{O}_{\sigma_{s_{1}}}=\mathbf{0}
$$

And yet, according to Proposition 3.3 in [6], $\mathcal{R}\left(\mathcal{O}_{\sigma_{s_{2}}} \mathcal{O}_{\sigma_{s 2}}^{\dagger}-\right.$ $\mathbf{1})=\operatorname{ker}\left(\mathcal{O}_{\sigma_{s_{2}}}\right)$. Therefore, since by definition $\operatorname{ker}\left(\mathcal{O}_{\sigma_{s_{2}}}\right)=$ $\Omega_{\sigma_{s_{2}}}$, (18) implies that $\Omega_{\sigma_{s_{2}}} \mathcal{O}_{\sigma_{s_{1}}}=\mathbf{0}$. That completes the proof.

2) Discernability:

Proposition 7: For system (1), discernability and (0)Backward Discernability (that is Backward Discernability) are not fulfilled.

Proof 6: Consider the two particular sequences $\sigma_{s_{1}}=$ $\sigma_{s_{[k-h, k-1]}} i(i \in \mathcal{J})$ and $\sigma_{s_{2}}=\sigma_{s_{[k-h, k-1]}} j(j \in \mathcal{J})$. For (3), the matrix $C_{\sigma(k)}$ does not depend on $\sigma(k)$. Therefore, the matrices $\mathcal{O}, T$, and $T^{\prime}$ defined as in (5) only depend on $\sigma_{s_{[k-h, k-1]}}$. Consequently, $T_{\sigma_{s_{1}}}=T_{\sigma_{s_{2}}}$ and $T_{\sigma_{s_{1}}}^{\prime}=T_{\sigma_{s_{2}}}^{\prime}$, which implies that (9) is not fulfilled.

Besides, since (0)-Backward Discernability is a special case of discernability, since Backward Discernability is not fulfilled, discernability is not fulfilled either. That completes the proof.

Since neither discernability nor (0)-Backward Discernability are satisfied, the whole sequence $\sigma_{s[k-h, k]}$ cannot be estimated. Let us focus on the less restrictive property, namely the $(\eta, \omega)$-discernability. We recall that such a property reflects the ability of discriminating, and so estimating, the subsequences $\sigma_{[k-h+\eta, k-\omega]}^{*}$.

3) $(\eta, \omega)-D i s c e r n a b i l i t y:$

Proposition 8: System (1) is not $(\eta=0, \omega=1)-$ discernible.

Proof 7: $(0,1)$-discernability is not satisfied if and only if neither (10) nor (11) in Proposition 2 is satisfied.

Let us consider the two particular sequences $\sigma_{s_{1}}=$ $(0,0)(0,0) \cdots(0,0)$ and $\sigma_{s_{2}}=(1,0)(0,0) \cdots(0,0)$ of length $h+1$. Let us note that the condition $\sigma_{s_{1}[k-h, k-1]} \neq$ $\sigma_{s_{2}[k-h, k-1]}$, as required in Proposition 2, is well satisfied. The respective observability matrices denoted $\mathcal{O}_{\sigma_{s_{1}}}$ and $\mathcal{O}_{\sigma_{s_{2}}}$ read

$$
\begin{aligned}
\mathcal{O}_{\sigma_{s_{1}}}= & {\left[\begin{array}{c}
C \\
C A_{(0,0)} \\
\vdots \\
C A_{(0,0)}{ }^{h}
\end{array}\right]=\left[\begin{array}{cccc}
\mathcal{C} & \mathbf{0} & \cdots & \mathbf{0} \\
\mathcal{C}(\mathcal{A}+\mathcal{G}) & \mathbf{0} & \cdots & \mathbf{0} \\
\mathcal{C}(\mathcal{A}+\mathcal{G})^{2} & \mathbf{0} & \cdots & \mathbf{0} \\
\vdots & \vdots & & \vdots \\
\mathcal{C}(\mathcal{A}+\mathcal{G})^{h} & \mathbf{0} & \cdots & \mathbf{0}
\end{array}\right] } \\
\mathcal{O}_{\sigma_{s_{2}}}= & {\left[\begin{array}{c}
C \\
C A_{(1,0)} \\
\vdots \\
C A_{(0,0)}{ }^{h-1} A_{(1,0)}
\end{array}\right] }
\end{aligned}
$$

$$
\mathcal{O}_{\sigma_{s_{2}}}=\left[\begin{array}{ccccc}
\mathcal{C} & \mathbf{0} & \mathbf{0} & \cdots & \mathbf{0} \\
\mathcal{C} \mathcal{A} & \mathcal{C} \mathcal{G} & \mathbf{0} & \cdots & \mathbf{0} \\
\mathcal{C}(\mathcal{A}+\mathcal{G}) \mathcal{A} & \mathcal{C}(\mathcal{A}+\mathcal{G}) \mathcal{G} & \mathbf{0} & \cdots & \mathbf{0} \\
\vdots & \vdots & \vdots & & \vdots \\
\mathcal{C}(\mathcal{A}+\mathcal{G})^{h-1} \mathcal{A} & \mathcal{C}(\mathcal{A}+\mathcal{G})^{h-1} \mathcal{G} & \mathbf{0} & \cdots & \mathbf{0}
\end{array}\right]
$$

Denote with $V_{1}$ the first column of $\mathcal{O}_{\sigma_{s_{1}}}$, and $V_{2}, V_{2}^{\prime}$ the first and the second column of $\mathcal{O}_{\sigma_{s_{2}}}$ respectively. It is clear that $V_{1}=V_{2}+V_{2}^{\prime}$. As a result,

$$
\operatorname{rank}\left(\left[\begin{array}{ll}
\mathcal{O}_{\sigma_{s_{1}}} & \mathcal{O}_{\sigma_{s_{2}}}
\end{array}\right]\right)=\operatorname{rank}\left(\mathcal{O}_{\sigma_{s_{2}}}\right)
$$

According to Proposition 6, it is equivalent to

$$
\Omega_{\sigma_{s_{2}}} \mathcal{O}_{\sigma_{s_{1}}}=\mathbf{0}
$$

On the other hand, since $D_{\sigma(k)}, B_{\sigma(k)}$ and $E_{\sigma(k)}$ do not depend on $\tau(k)$, it holds that $D_{(0,0)}=D_{(1,0)}, B_{(0,0)}=$ $B_{(1,0)}$ and $E_{(0,0)}=E_{(1,0)}$. As a result, from the definitions of $T$ and $T^{\prime}$ given in (5), we have that $T_{\sigma_{s_{1}}}=T_{\sigma_{s_{2}}}$ and $T_{\sigma_{s_{1}}}^{\prime}=T_{\sigma_{s_{2}}}^{\prime}$ which implies that

$$
\Omega_{\sigma_{s_{2}}}\left(\left(T_{\sigma_{s_{1}}}-T_{\sigma_{s_{2}}}\right) u_{k-h, k}+\left(T_{\sigma_{s_{1}}}^{\prime}-T_{\sigma_{s_{2}}}^{\prime}\right)\right)=\mathbf{0}
$$

As a result, from (20) and (21), it holds that Proposition 2 is not satisfied and the sequences $\sigma_{s_{1}}$ is not $(0,1)$-discernible from $\sigma_{s_{2}}$. That completes the proof.

\section{4) ( $\lambda)$-Backward Discernability:}

Proposition 9: If $U_{k}$ remains unchanged between two consecutive discrete times $k$ and $k+1$, system (1) is not $(\lambda=1)$-Backward Discernible.

Proof 8: Let us consider the sequences $\sigma_{s_{3}}=\sigma^{h-\lambda} i(0,0)$ and $\sigma_{s_{4}}=\sigma^{h-\lambda} j(0,0)$ of length $h+1$ with $\sigma^{h-\lambda}=$ $(0,0)(0,0) \cdots(0,0), i=(0,0)$ and $j=(0,1)$. It suffices to show that conditions (13) and (14) of Proposition 4 are not satisfied for $\sigma_{s_{3}}$ and $\sigma_{s_{4}}$.

For both sequences $\sigma_{s_{3}}$ and $\sigma_{s_{4}}$, it holds that $\tau(k)=0$ for all $k$. Since the observability matrix $\mathcal{O}_{\sigma_{[k-h, k]}}$ exclusively depends on $\tau(k), \mathcal{O}_{\sigma_{s_{3}}}=\mathcal{O}_{\sigma_{s_{4}}}$ and then $\operatorname{ker}\left(\mathcal{O}_{\sigma_{s_{3}}}\right)=$ $\operatorname{ker}\left(\mathcal{O}_{\sigma_{s_{4}}}\right)$ which implies that

$$
\Omega_{\sigma_{s_{4}}} \mathcal{O}_{\sigma_{s_{3}}}=\mathbf{0}
$$

On the other hand, it holds that $A_{(0,0)}=A_{(0,1)}, D_{(0,0)}=$ $D_{(0,1)}$ and $E_{(0,0)}=E_{(0,1)}$ since $A_{\sigma(k)}, D_{\sigma(k)}$ and $E_{\sigma(k)}$ do not depend on $\tau^{\prime}(k)$. From the definitions of $T$ and $T^{\prime}$ given in (5), we have that $T_{\sigma_{s_{3}}}^{\prime}=T_{\sigma_{s_{4}}}^{\prime}$. Thus, (9) becomes

$$
\Omega_{\sigma_{s_{4}}}\left(\left(T_{\sigma_{s_{3}}}-T_{\sigma_{s_{4}}}\right) u_{k-h, k}\right) \neq \mathbf{0}
$$

with

$$
\begin{aligned}
T_{\sigma_{s_{3}}}-T_{\sigma_{s_{4}}}= & {\left[\begin{array}{cccc}
\mathbf{0} & & \mathbf{0} & \mathbf{0} \\
\vdots & \vdots & & \vdots \\
\mathbf{0} & & \mathbf{0} & \mathbf{0} \\
\mathbf{0} & C\left(B_{(0,0)}-B_{(0,1)}\right) & \mathbf{0}
\end{array}\right] } \\
= & {\left[\begin{array}{cccc}
\mathbf{0} & \mathbf{0} & \mathbf{0} & \mathbf{0} \\
\vdots & \vdots & \vdots & \vdots \\
\mathbf{0} & \mathbf{0} & \mathbf{0} & \mathbf{0} \\
\mathbf{0} & \mathcal{C B} & -\mathcal{C B} & \mathbf{0}
\end{array}\right] }
\end{aligned}
$$


Substituting $T_{\sigma_{s_{3}}}-T_{\sigma_{s_{4}}}$ into (23) yields

$$
\Omega_{\sigma_{s_{4}}}\left[\mathbf{0} \quad \mathcal{C B} U_{k-1}-\mathcal{C B} U_{k-2}\right]^{T} \neq \mathbf{0}
$$

Hence, if $U_{k-1}=U_{k-2}$, from (22) and (24), we infer that neither (13) nor (14) of Proposition 4 is satisfied and the sequence $\sigma_{s_{3}}$ is not (1)-Backward Discernible from $\sigma_{s_{4}}$. That completes the proof.

\section{EXAMPLE}

Let us consider (1) with the particular setting

$$
\begin{gathered}
\mathcal{A}=\left[\begin{array}{cc}
-0.21 & 0.1 \\
0 & 1
\end{array}\right], \mathcal{G}=\left[\begin{array}{cc}
0.25 & 0 \\
0 & -0.21
\end{array}\right], \\
\mathcal{B}=\left[\begin{array}{cc}
1.5 & 0 \\
1 & 0.8
\end{array}\right], \mathcal{C}=\left[\begin{array}{cc}
1 & 3 \\
2.5 & 0
\end{array}\right], \\
\mathcal{E}=\left[\begin{array}{c}
1 \\
0.3
\end{array}\right], \mathcal{D}=\mathbf{0}
\end{gathered}
$$

The delays fulfill $\tau(k) \in\{0,1,2\}(\alpha=2), \tau^{\prime}(k)=0$ for all $k\left(\alpha^{\prime}=0\right)$.

This linear delayed discrete-time system is rewritten into the form (3) with

$$
x_{k}=\left[\begin{array}{c}
X_{k} \\
X_{k-1} \\
X_{k-2}
\end{array}\right], \quad u_{k}=U_{k}, \quad y_{k}=Y_{k}
$$

and the switching rule $\sigma$ is defined as $\sigma: k \in \mathbb{N} \rightarrow \sigma(k) \in \mathcal{J}$ with $J=(\alpha+1)\left(\alpha^{\prime}+1\right)=3$ as the number of modes. The corresponding state space matrices read

$$
\begin{gathered}
A_{1}=\left[\begin{array}{ccc}
\mathcal{A}+\mathcal{G} & \mathbf{0} & \mathbf{0} \\
\mathbf{1} & \mathbf{0} & \mathbf{0} \\
\mathbf{0} & \mathbf{1} & \mathbf{0}
\end{array}\right], A_{2}=\left[\begin{array}{ccc}
\mathcal{A} & \mathcal{G} & \mathbf{0} \\
\mathbf{1} & \mathbf{0} & \mathbf{0} \\
\mathbf{0} & \mathbf{1} & \mathbf{0}
\end{array}\right], \\
A_{3}=\left[\begin{array}{ccc}
\mathcal{A} & \mathbf{0} & \mathcal{G} \\
\mathbf{1} & \mathbf{0} & \mathbf{0} \\
\mathbf{0} & \mathbf{1} & \mathbf{0}
\end{array}\right], B_{i}=\left[\begin{array}{l}
\mathcal{B} \\
\mathbf{0} \\
\mathbf{0}
\end{array}\right], E_{i}=\left[\begin{array}{l}
\mathcal{E} \\
\mathbf{0} \\
\mathbf{0}
\end{array}\right], \\
C_{i}=\left[\begin{array}{lll}
\mathcal{C} & \mathbf{0} & \mathbf{0}
\end{array}\right], D_{i}=0 \quad \forall i \in\{1,2,3\}
\end{gathered}
$$

where $\sigma(k)=1$ is associated to $\left(\tau, \tau^{\prime}\right)=(0,0), \sigma(k)=2$ is associated to $\left(\tau, \tau^{\prime}\right)=(1,0)$, and $\sigma(k)=3$ is associated to $\left(\tau, \tau^{\prime}\right)=(2,0)$.

The smallest detection horizon $h$ which satisfies (7) is $h=3$.

- Initial estimation

The property of $(\eta, \omega)$-discernability is checked using (10) since (11) is never verified because $B_{1}=B_{2}$ and $E_{1}=E_{2}$. After having performed the test, it holds that (10) is satisfied for $\eta=2$ and $\omega=1$ which means that $(2,1)$-discernability is fulfilled.

\section{- Permanent estimation}

Since (14) is never verified because $B_{1}=B_{2}, E_{1}=E_{2}$ and $C_{1}=C_{2}$, conditions (13) are checked to test $(\lambda)$-Backward Discernability. It holds that (13) is fulfilled for $\lambda=1$. Therefore, the permanent estimation can be performed by the detector which delivers the active mode $\sigma^{*}(k-1)$ for $k>h+\omega+\eta-\lambda-1=4$. The detector successfully recovers the unknown delay as depicted on Figure 1 (bottom).
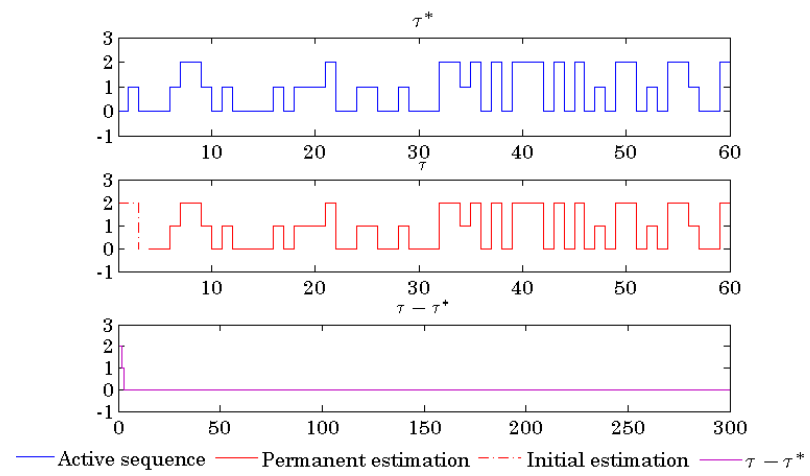

Fig. 1. Top: active sequences $\tau^{*}$. Middle: reconstructed sequences $\tau$. Bottom: error $\tau-\tau^{*}$

\section{CONCLUSION}

We have presented an approach to estimate time-varying delays of discrete-time linear and affine systems. The problem has been turned into a mode detection problem for switched affine systems. Conditions on discernability have been particularized to this special context.

\section{Acknowledgement}

This work was partially supported by Research Grants ANR-13-INSE-0005-01 from the Agence Nationale de la Recherche.

\section{REFERENCES}

[1] A. Alessandri, M. Baglietto, and G. Battistelli. Receding-Horizon Estimation for Switching Discrete-Time Linear Systems. IEEE Trans. on Automatic Control, 50(11), 2005.

[2] M. Babaali and M. Egerstedt. Observability of Switched Linear Systems. Hybrids Systems: Comput. and Control, pages 48-63, 2004.

[3] M. Babaali and M. Egerstedt. Asymptotic Observers for DiscreteTime Switched Linear Systems. In Proceedings of the 16th IFAC World Congress, Czech Republic, 2005.

[4] M. Baglietto, G. Battistelli, and L. Scardovi. Active mode observability of switching linear systems. Automatica, 43(11):1442-1449, 2007.

[5] M. Baglietto, G. Battistelli, and L. Scardovi. Active mode observation of switching linear systems based on set-valued estimation on the continuous state. Int. J. Robust Nonlinear Cont., 19:1521-1540, 2009.

[6] J. C. A. Barata and M. S. Hussein. The Moore-Penrose Pseudoinverse: A Tutorial Review of the Theory. Brazilian Journal of Physics, 42:146-165, 2012.

[7] L. Belkoura, J-P. Richard, and M. Fliess. Parameters estimation of systems with delayed and structured entries. Automatica, 45(5):1117$1125,2009$.

[8] S. Bjorklund and L. Ljung. A Review of Time-Delay Estimation Techniques. In Proc. 42nd IEEE Conf. on Decision and Cont., 2003.

[9] E. A. Domlan, J. Ragot, and D. Maquin. Switching Systems Mode Estimation using a Model-based Diagnosis Method. In 8th Conference on Diagnostics of Processes and Systems, Slubice, Poland, 2007.

[10] M. Halimi, G. Millérioux, and J. Daafouz. A hybrid approach for the estimation of time-varying delays of discrete-time systems. IFAC Joint conference, Grenoble, France, February 4-6, 2013.

[11] L. Hetel, J. Daafouz, and C. Iung. Equivalence Between the LyapunovKrasovskii Functional Approach for Discrete Delay Systems and the Stability Conditions for Switched Systems. Nonlinear Analysis: Hybrids Systems, pages 697-705, 2008.

[12] G. Nikolakopoulos, A. Panousopoulou, A. Tzes, and J. Lygeros. MultiHopping Induced Gain Scheduling for Wireless Networked Controlled Systems. In Proceedings 44th IEEE Conference on Decision and Control (CDC'05), Seville, 2005.

[13] R. Vidal, A. Chiuso, and S. Soatto. Observability and Identifiability of Jump Linear Systems. In In Proc. of IEEE Conference on Decision and Control, 2002. 